\title{
Evaluation of changes in dry and wet precipitation extremes in warmer climates using a passive water vapor modelling approach
}

\author{
Marie-Pier Labonté and Timothy M. Merlis \\ McGill University, Montreal, Quebec, Canada
}

\begin{abstract}
Hydroclimatic extremes, such as heavy daily rainfall and dry spells, are expected to intensify under anthropogenic warming. Often, these changes are diagnostically related to thermodynamic increases in humidity with warming. Here, we develop a framework that uses an on-line calculation of the thermodynamically induced changes of the full precipitation distribution with warming in an idealized moist atmospheric general circulation model. Two water vapor variables, the standard active one and an additional passive one (i.e., no latent heat release when condensation occurs), are advected by the resolved circulation. The passive water vapor is thermodynamically perturbed by modifying the saturation specific humidity used in the calculation of its condensation tendency and surface evaporation. The difference between the precipitation of the perturbed passive water vapor relative to the control one corresponds to the thermodynamic component of precipitation change, which can be evaluated for the entire distribution. Here, we evaluate wet and dry extremes. Our simulations have tropical increases and higher latitude decrease of dry spells' length (defined as the maximum consecutive dry days), as found in the zonal-mean of comprehensive models. This simulated thermodynamically induced intensification of dry spells in the tropics arises from the decreased contrast between sea surface temperature and surface air temperature with warming. The simulated increase in heavy daily rainfall (e.g., the 99.9th percentile of the daily precipitation distribution) at all latitudes differs modestly from a previous theory that assumes moist adiabatic stratification and increased warming aloft slightly damps the simulated increase, as the theory suggests.
\end{abstract}

\section{Introduction}

Climate models' projections of the future daily precipitation distribution have an intensification of hydroclimatic extremes under global warming. Understanding the projected characteristics' changes of both dry and wet extreme events, such as their intensity and frequency of occurrence, is crucial to grasp the full extent of their future societal impacts. Dry and wet hydroclimatic extremes are often defined as dry spells and heavy daily rainfall, respectively. While regions expected to have increased mean precipitation (deep tropics and mid-to-high latitudes) generally have shorter dry spells and regions expected to have decreased mean precipitation (subtropics) have longer dry spells in future projections, heavy daily rainfall is projected to increase almost everywhere (Dai et al. 2018; Fischer et al. 2013; Pfahl et al. 2017; Sillmann et al. 2013). Dry and wet extremes do not have identical constraints, as changes in both extremes with warming are driven by combinations of dynamic processes (changes in the large-scale circulation, convective vertical velocities, and eddies) and thermodynamic processes (the dependence of atmospheric water vapor on temperature, in particular).

It is well established that thermodynamically induced rainfall changes are linked to atmospheric water vapor changes. Water vapor changes follow Clausius-Clapeyron (CC) scaling, the quasi-exponential relation between saturation specific humidity and temperature, with a global value of $6-7 \%$ per degree of warming, because changes in

\footnotetext{
Corresponding author: Marie-Pier Labonté, mariepier.labonte@mail.mcgill.ca
}

relative humidity are small (Trenberth et al. 2003). Allen and Ingram (2002) proposed that extreme precipitation changes follow the CC scaling based on the premise that an extreme event empties the air parcel of all its water vapor. The thermodynamic intensification of extreme precipitation is actually most likely to be at a sub-CC rate (Chen et al. 2011; O'Gorman and Schneider 2009; Pall et al. 2007), due to the relation between extreme precipitation and the moist-adiabatic derivative of saturation specific humidity. The latter increases at a slower rate than the CC scaling, due to the offsetting effect of moist adiabatic warming (O'Gorman and Schneider 2009). Thermodynamic changes have also been invoked for the dry tail of the distribution of precipitation: if the mean precipitation increases more weakly (e.g., 2-3\% per degree of warming) than the specific humidity, this suggests there must be compensating drying in parts of the precipitation distribution should the increase in extremes exceed the mean. One of the contributions of this research is to thoroughly quantify thermodynamic changes across the distribution of precipitation.

Beyond thermodynamic changes, there is a substantial body of studies that examine the dynamic contribution to projected changes in heavy daily precipitation. The expected dynamical poleward shift of the circulation under global warming may have a large contribution to extreme precipitation intensity and frequency in the midlatitudes (Lu et al. 2014). In addition, the strengthening of midtropospheric vertical velocities can amplify heavy rainfall intensity. Some studies use the quasi-geostrophic (QG) omega equation to decompose the vertical velocity changes 
into a dry component- the textbook ageostropic terms in QG theory-and a moist component-arising from latent heat release (e.g., Li and O'Gorman 2020). The moist component reveals that the latent heat released during an extreme event is linked to changes in moist static stability (a latent heat feedback) because a stability closer to moist adiabatic leads to an increased pressure depth of the upward motion ( $\mathrm{Li}$ and O'Gorman 2020). The QG omega equation's moist component also reveals that the nonlinear relation of the diabatic-heating feedback with increased water vapor explains the larger magnitude intensification in moistening regions (Nie et al. 2020). Heavier wet extremes are also projected in drying regions, such as the subtropics, albeit with large uncertainties (Pfahl et al. 2017; Norris et al. 2020). While weaker extreme ascent can result of increased horizontal scale of ascent and increased stability in the subtropics (Tandon et al. 2018), there is also evidence of an amplification of extreme ascent associated with the heaviest rainfall events, though this may be damped in certain regions due to the Hadley cell's poleward expansion (Norris et al. 2020). While typical climate model resolutions rely on convective parameterization, cloud-resolving model (CRM) have also been used to investigate the future vertical velocities changes associated to extreme precipitation. There is evidence that vertical velocities' peak shifts upward with warming (Abbott et al. 2020; Muller et al. 2011), and this influences the relevant temperatures where condensation occurs. As for droughts/dry spells, it has been shown that a simulated increase in dry extremes is the result of a modest strengthening of ascent in convective regions, and the associated expansion of the surrounding dry areas (Lintner et al. 2012). It has been linked to the upped-ante mechanism proposed by Neelin et al. (2003), by which decreases in rainfall result from of an increase in the required surface boundary layer moisture for convection to occur. In brief, dynamical changes, via convective velocities in low latitudes and synoptic scale eddies in the mid-latitudes, are important to precipitation extremes.

We aim to disentangle the thermodynamically induced changes from the dynamic-induced changes across the full probability distribution of daily precipitation, simultaneously. Chen et al. (2019) developed a quantile-conditional moisture budget which can be applied to the whole daily precipitation probability distribution function. They were able to obtain the thermodynamic and dynamic components for high percentile daily rainfall. They demonstrate the "wet get wetter" mechanism controls extreme precipitation increase, where the amplification is linked to an increased gross moisture stratification controlled by lowertropospheric moisture changes. We use a different method to obtain the relative importance of thermodynamic effects over the whole probability distribution of daily precipitation, disentangled from dynamical influences. Our methodological approach involves the implementation of a passive water vapor within the GCM, which follows the dynamical flow without interacting with it. This method is similar to Grabowski (2014), who used two set of thermodynamic variables to study microphysical schemes impact on convection, and isotope-enabled GCMs (e.g., Colose et al. 2016; Lee et al. 2009), where multiple water isotope tracers following the same dynamical flow but with different temperature-dependent sources and sinks, to study past climates. Here, we use a passive water vapor as a direct measure of the thermodynamic precipitation change from different temperature perturbations that result from warming due to a strengthened atmospheric greenhouse, akin to increased carbon dioxide concentration.

We evaluate the thermodynamically induced changes for both dry and wet extremes under global warming. The passive water vapor framework, which can be used for both individual precipitation events and climatological changes, simulates a substantial thermodynamically induced precipitation decrease of dry and wet days in the subtropics and of dry days in mid-latitudes. We show an important role for the tropical decrease in the air-sea surface temperature contrast with warming, which damps tropical surface evaporation increases, in simulating a thermodynamically induced amplification of dry-spell length and of increased warming aloft in the tropics in simulating a small thermodynamic damping of the intensification of extreme precipitation.

\section{Methods \\ a. Idealized General Circulation Model}

We use the GFDL gray-radiation aquaplanet moist GCM (Frierson et al. 2006), with a T85 truncation in the spectral dynamical core $\left(\sim 1.4^{\circ}\right.$ horizontal resolution $)$ and 30 sigma $(\sigma)$ vertical levels. This is an extensively analyzed, idealized "rung" of the hierarchy of climate models (Held 2005; Jeevanjee et al. 2017; Maher et al. 2019). The sigma coordinate is the pressure coordinate normalized by its surface value $\left(\sigma=p / p_{s}\right)$. The model does not include any convection scheme (more discussion of that choice is provided in the following section). There is no transport of condensates: they immediately precipitate out. Surface precipitation is then simply the mass-weighted vertical integral of the large-scale condensation tendency of water vapor. The aim of our research study is to provide an analysis of the changes of daily precipitation statistics with warming induced by an increased greenhouse gas concentration.

We perform a simulation where the atmospheric optical depth is increased 1.4 times (we will refer to this simulation as "warmed" from now on), following O'Gorman and Schneider (2008). Both the control and warmed simulation have zonally symmetric slab ocean lower boundary conditions that simulate an energetically consistent surface temperature distribution. The difference between the warmed and control simulation is used to define the total 
(combined dynamic and thermodynamic) change in precipitation. The change in time- and zonal-mean temperature is shown in Fig. 1a and has substantial structural similarity to comprehensive GCMs: enhanced warming in the upper tropical troposphere and polar amplified surface warming that is associated with a lower-troposphere enhanced warming. This temperature change field plays a key role in the passive water vapor approach, described next, that we use to disentangle the effects atmospheric thermodynamic changes and dynamic changes have on daily precipitation statistics.

\section{b. Passive water vapor approach}

There are four existing modelling approaches with common aspects to ours:

1. The "piggy-backing" approach of Grabowski (2014) and Grabowski (2015), who examined microphysical impacts on convection. They use two sets of thermodynamic variables affected each by a different microphysical scheme. One set of the thermodynamic variables determines the dynamical flow, while the other one is "piggy-backing". This isolates microphysical changes (e.g., changes in cloud condensation nuclei from increased aerosols) without the associated circulation-related changes that occur in time-dependent nonlinear atmospheric models.

2. Regional tagging of additional water vapor tracer variables (e.g., Bosilovich and Schubert 2002), where passive water vapor tracers follow the same Eulerian moisture tendency equation, but are "tagged" to reveal the region where surface evaporation occurred.

3. Isotope-enabled GCMs, as described in the introduction.

4. Idealized dry GCMs with passive hydrological cycles (e.g., Galewsky et al. 2005; Ming and Held 2018), in which a standard dry dynamical core with diabatic forcing (Held and Suarez 1994) that is independent of the simulated water vapor condensation is used to advect water vapor and it is subject to condensation when it is supersatured.

We perform simulations with two sets of water vapor variables. There is the standard prognostic water vapor variable (referred to as control water vapor) plus a second one tagging along without interacting with the simulated prognostic atmospheric state variables (referred to as passive water vapor). In our study, the two water vapor variables also follow the same prognostic equation for moisture (equation 1) without interacting with each other at any time

$$
\partial_{t} q=\partial_{t} q_{\text {cond }}+\partial_{t} q_{\text {evap }}+\partial_{t} q_{\text {diff }}-\mathbf{u} \cdot \nabla q \text {. }
$$

The moisture sink and sources for both water vapor variables are respectively the large-scale condensation tendency $\partial_{t} q_{\text {cond }}$, the surface evaporation tendency $\partial_{t} q_{\text {evap }}$, and the turbulent vertical diffusion tendency (sub-grid scale boundary layer turbulence, in particular) $\partial_{t} q_{\text {diff } f}$. The transport of water vapor is managed by the tracer advection $\mathbf{u} \cdot \nabla q$. Both the control and the passive water vapor are advected by the same control resolved-scale circulation $\mathbf{u}$. The passive water vapor departs from the control water vapor only in the case where we perturb the passive variable. The choice of the term "passive" refers to the absence of latent heat released when condensation occurs. This means there is it does not feedback on the prognostic atmospheric state variables (temperature, surface pressure, active specific humidity, or winds). This way, when the passive water vapor is thermodynamically perturbed, it is completely disentangled from dynamicallyinduced changes.

We perturb the passive water vapor to perform a direct "on-line" calculation (within the GCM) of the thermodynamic effect of an increased greenhouse absorbers (akin to atmospheric $\mathrm{CO}_{2}$ concentration) on the daily precipitation statistics. The perturbation is purely thermodynamic, meaning it is linked to atmospheric water vapor changes due to temperature changes. Atmospheric temperature perturbations modify the atmospheric saturation specific humidity (i.e., the atmosphere's capacity to hold water vapor) which alters principally when/where the resolvedscale condensation from supersaturation occurs. The surface evaporation (i.e., the amount of moisture input in the atmosphere) is altered by temperature changes in the lowest atmospheric level and at the surface. The vertical diffusion tendency and the advection term are not directly modified by a pure thermodynamic perturbation; the former is mostly driven by sub-grid scale turbulent motions, while the latter is tied to the large-scale circulation. It is important to note that the vertical diffusivity is dependent on the vertical gradient of specific humidity, which differs between the passive water vapor and the active one. This means that the vertical diffusion tendency does not remain unchanged for the thermodynamically perturbed passive water vapor. We add a specified time-mean temperature perturbation $\Delta \bar{T}(\sigma, \phi)$ to the GCM-simulated temperature field $T^{o}(t, \sigma, \phi)$ used to calculate the temperature-dependent large-scale condensation and the surface evaporation tendencies of the passive water vapor. The prognostic equation for the perturbed passive water vapor is now

$$
\partial_{t} q=\partial_{t} q_{c o n d}\left(q, T^{o}+\Delta \bar{T}, p^{o}\right)+\partial_{t} q_{\text {evap }}\left(q, T^{o}+\Delta \bar{T}\right)+\partial_{t} q_{\text {diff } f}\left(q, \mathbf{u}^{o}\right)-\mathbf{u}^{o} \cdot \nabla q
$$


where the superscript $o$ denotes to control-climate state variables with time fluctuations and $\overline{(\cdot)}$ denotes time-mean. The control water vapor and the control resolved-scale circulation are unchanged; only the source and sink of the passive water vapor are modified by this temperature perturbation (equation 2). Given that condensates immediately precipitate out in the GCM, the resulting changes in the large-scale condensation directly provides the simulated surface precipitation response to the specified thermodynamic forcing $\Delta \bar{T}(\sigma, \phi)$.

It is important to mention that we did not include the convection tendency in the moisture equation because of an important issue with how the convection schemes (e.g., Frierson 2007) and the passive water vapor interact. Because the latter always feels a temperature that is warmer than the environment temperature, it has the potential to be in constant conditionally unstable state, depending on the vertical structure of the perturbation $\Delta \bar{T}$. This is particularly a concern in the deep tropics. We choose to turn off the convection scheme even though this degrades the control simulation and can affect the response to climate change. As it becomes increasingly feasible to run GCMs near convection-resolving resolutions (or cloud-system resolving models in near global domains), we did not pursue strategies to ameliorate this concern. We also note that isotope-enabled GCMs face issues in how convection schemes deal with the different isotopes.

\section{c. Decomposing the temperature perturbation}

We design four different experiments using different structures (latitudinal and vertical) for the temperature perturbation $\Delta \bar{T}(\sigma, \phi)$, with the intent of isolating the relative importance of different well known features of warming that arise from a stronger atmospheric greenhouse.

In the first experiment ("Full Structure", Figure 1a), the temperature change field is the time- and zonal-mean temperature difference between the control and the warmed simulations (with the perturbation described in section 2a). In the second experiment ("Vertically Uniform", Figure $1 \mathrm{~b}$ ), the temperature change is a simplification of the first one: We remove the vertical structure from the temperature perturbation by replicating the lowest atmospheric level temperature change throughout the whole atmospheric column at each latitude. This preservers the meridional structure of warming (e.g., polar amplification) and the change between the surface temperature and surface-air temperature. In the third experiment ("Uniform", Figure 1c), there is a homogeneous temperature perturbation at all latitudes, all vertical levels, and at the surface. This constant temperature change is set at $\Delta \bar{T}(\sigma, \phi)=3 \mathrm{~K}$, but the results do not depend sensitively on the magnitude. We performed an experiment with a homogeneous temperature of $\Delta \bar{T}(\sigma, \phi)=6 \mathrm{~K}$, which had similar normalized results.
In the fourth and last experiment ("Moist-adiabat", Figure 1d), we assume the temperature perturbation follows a moist-adiabatic profile. The moist-adiabatic profiles are calculated based on the simulated surface temperature, surface relative humidity, and surface pressure of the control and the warmed simulations, the difference between these gives the moist-adiabatic temperature perturbation. The moist-adiabatic temperature change simulation is used to compare to existing scaling theory for wet extremes, so we only present the results in section $3 \mathrm{e}$. In these four experiments, the time-independent temperature perturbation is applied only to the passive water vapor (see equation 2), and the control water vapor stays unchanged.

\section{Results}

\section{a. Thermodynamic precipitation change of an individual extreme event}

Figure 2a shows a map of the daily precipitation for an individual extreme event, located at $40-50^{\circ} \mathrm{N}$ lat, $60-75^{\circ}$ lon. This is a 99.9th percentile event for $45^{\circ} \mathrm{N}$ latitude, and we can see the storm structure of a midlatitude cyclone, as the pressure velocity at the $550 \mathrm{hPa}$ pressure level suggests (grey contour lines). Within the same GCM simulation, the result of the on-line calculation of precipitation arising from the condensation of the passive water vapor at the same time is shown in Figure $2 \mathrm{~b}$. The spatial pattern is similar, but the local maxima are generally enhanced, as one expects from increased humidity. We show this sample individual extreme event to highlight that the methodology we use here can readily be applied to individual storms, in addition to performing analysis of the climatological changes in the full distribution of precipitation that we present in what follows. Previous authors have analyzed individual tropical cyclones in models where both the humidity and circulation were free to evolve (Trenberth et al. 2007; Lackmann 2015; Emanuel 2017; Reed et al. 2020) and we are applying the modelling approach introduced here to idealized GCM simulations configurations with many tropical cyclones in forthcoming work.

\section{b. Daily precipitation distribution}

For a given simulation, we get the daily precipitation distribution for each latitude by aggregating the data for all longitude grid points (which takes advantage of the zonally symmetric boundary conditions to improve the statistics for rarer events by creating a much longer time series) and then sorting these values. The cumulative density function (CDF) of the control daily precipitation is shown in Figure 3 to provide an overview of the statistics of daily precipitation, from dry extremes to wet extremes, at all latitudes. For example, the value of the CDF's 10th percentile at a given latitude indicates that the $10 \%$ days with the lowest 

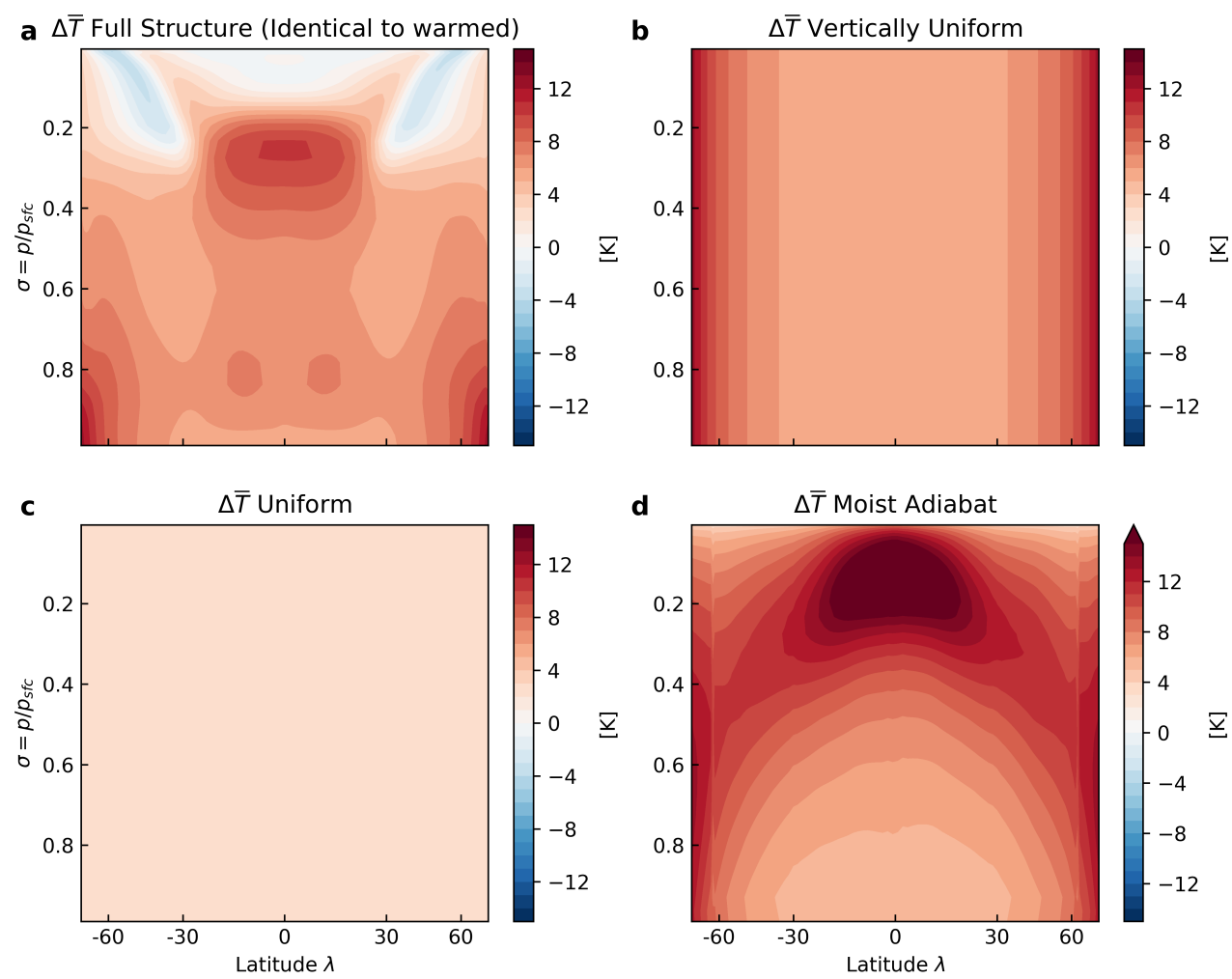

FIG. 1. Four panels showing each time-mean, zonal-mean temperature fields $\Delta \bar{T}(\lambda, \sigma)$ used to perturb the passive water vapor in the four experiments described in section 2c. (a) "Full Structure": atmospheric warming with vertical and latitudinal gradients resulting from an increased atmospheric $\mathrm{CO}_{2}$ concentration (taken from the warmed experiment). (b) "Vertically Uniform": atmospheric warming vertically uniform with latitudinal gradient, replication of the lowest atmospheric temperature change at all vertical levels for each latitudes. (c) "Uniform": homogeneous warming ( 3K). (d) "Moist-adiabat": atmospheric warming following a moist-adiabatic profile, which is calculated based on the simulated surface temperature, surface relative humidity and surface pressure (via the control and warmed simulations).

rainfall, including days with no precipitation, have a precipitation rate equal or less than this value and there are many latitudes where the 10th percentile is indeed zero.

In this study, we are specifically interested in the changes of the daily precipitation distribution with warming. We take the difference between the distributions for the control and the thermodynamically perturbed daily precipitation to obtain the simulated percentage change with warming of all daily precipitation's percentiles, for each latitude.

Figure 4a shows the precipitation change obtained via the difference between the active precipitation in the warmed and control simulation. This standard GCM precipitation change (using the difference of two simulations without passive water vapor hydrological cycles) includes both thermodynamic and dynamic-related precip- itation changes. In the midlatitudes, there is a precipitation decrease with warming at lower percentiles $(<40$ th $)$, while there is a precipitation increase with warming at higher percentiles $(>60$ th). This indicates a general tendency towards dry days getting drier and wet days getting wetter; there is a simulated shift of the CDF shape towards moderate-heavy rainfall events at the expense of low rainfall events. The white regions corresponds to the days with no rainfall in the control climate.

Figure $4 \mathrm{~b}$ shows the thermodynamically induced changes of the daily precipitation statistics calculated using the passive framework. The difference between the standard precipitation's total change and this thermodynamic change determined via the passive water vapor can be thought of as dynamic changes. We see that the ther- 


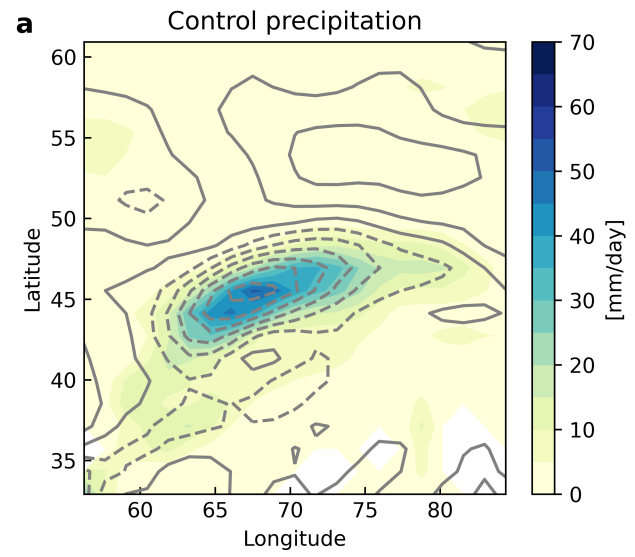

b

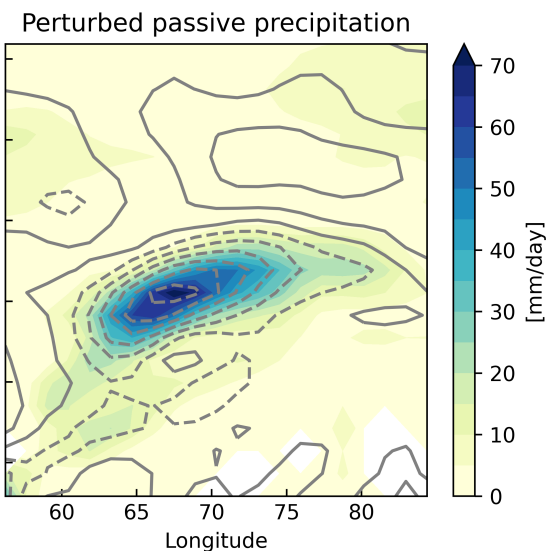

FIg. 2. A snapshot of an individual 99.9th rainfall event (at 45N) in the midlatitudes, result of the condensation of (a) the control standard water vapor, and (b) the thermodynamically-perturbed passive water vapor $(\Delta \bar{T}$ Full Structure, see Fig. 1a). Surface precipitation (colour-filled contours at $5 \mathrm{~mm} /$ day interval), and pressure velocity at $550 \mathrm{hPa}$ (grey contours at $0.1 \mathrm{~Pa} / \mathrm{s}$ interval, dashed for negative values (upward motion)).
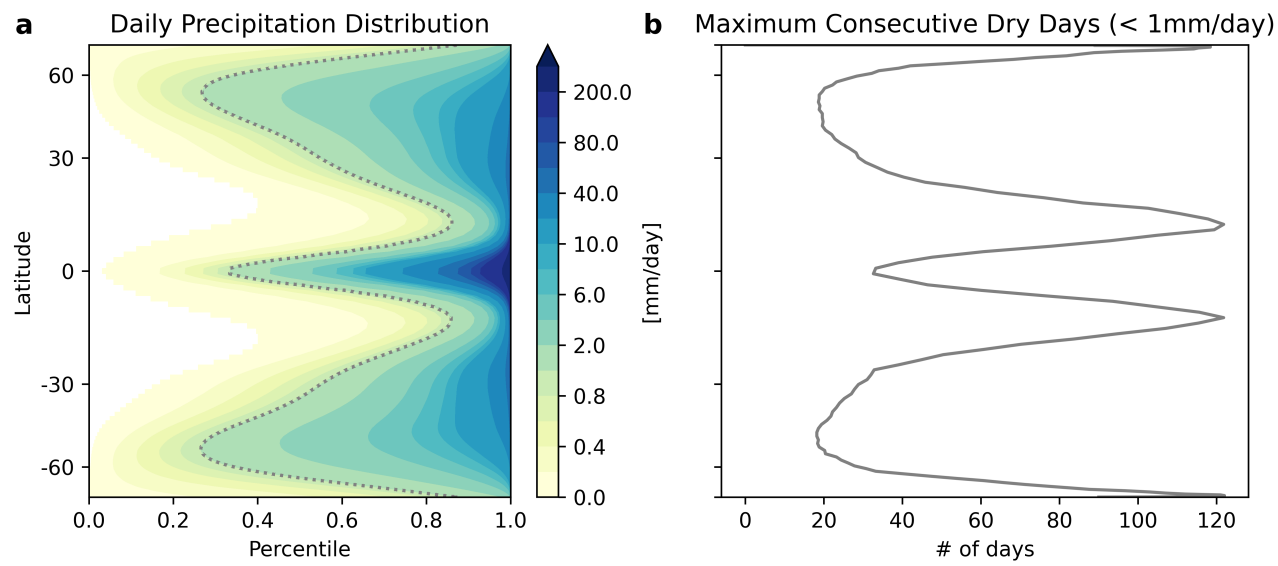

FIG. 3. (a) Daily precipitation distribution for the control climate, at all latitudes. Daily rainfall (colour-filled contours at pseudo$\log$ interval). The dotted grey line denotes the percentile at which a daily precipitation rate of $1 \mathrm{~mm} /$ day is found, our defined threshold between dry and wet days. The white area indicates the days without rainfall (0mm/day). (b) Climatology maximum consecutive dry days at each latitudes. The threshold between dry and wet days is set at $1 \mathrm{~mm} /$ day here.

modynamic change (Fig. 4b) captures an important part of the total changes (Fig. 4a) in the statistics of daily precipitation. Our simulation reproduces the large precipitation rate decrease in the subtropics across nearly all percentiles and the precipitation rate increase of wet days (higher percentiles) in the deep tropics, mid and high latitudes. The dry and wet extremes are analyzed in more detail in the next sections.

The effect of the vertical structure of the warming (see Fig. 1) on thermodynamic precipitation changes is eliminated in Figure $4 c$ (compare to $4 b$ ). The simulated precipitation rate decrease of the subtropical regions at lower percentiles does not depend strongly on the vertical structure of warming. The thermodynamic perturbation is further simplified to a homogeneous warming (Fig. 4d), by eliminating the horizontal structure (e.g., no polar amplification) and the temperature change difference between surface and lower atmospheric level (which modifies the evaporative surface flux of the passive water vapor). With this temperature perturbation, the simulated precipitation rate decrease is limited in the lower percentiles (30th-40th percentile) of the subtropics. In summary, the warming difference between the sea surface temperature and surface air temperature in a warmer climate is important to 
Changes in daily precipitation distribution
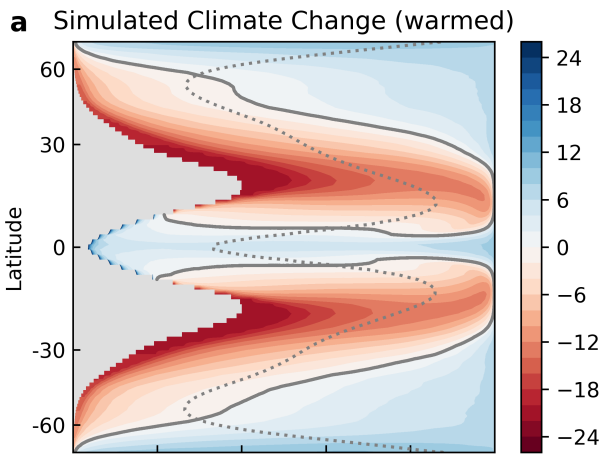

C

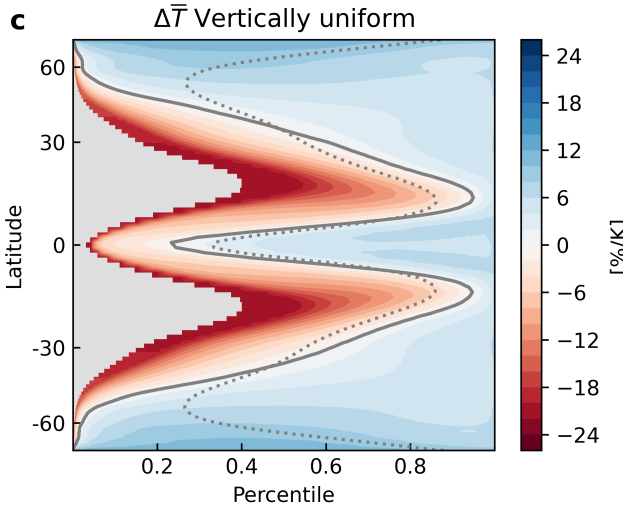

b

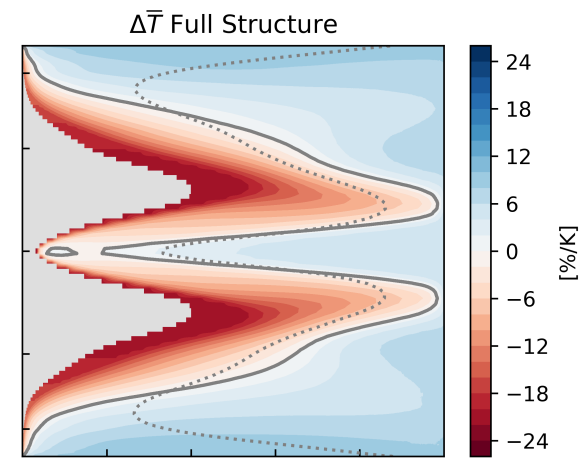

d

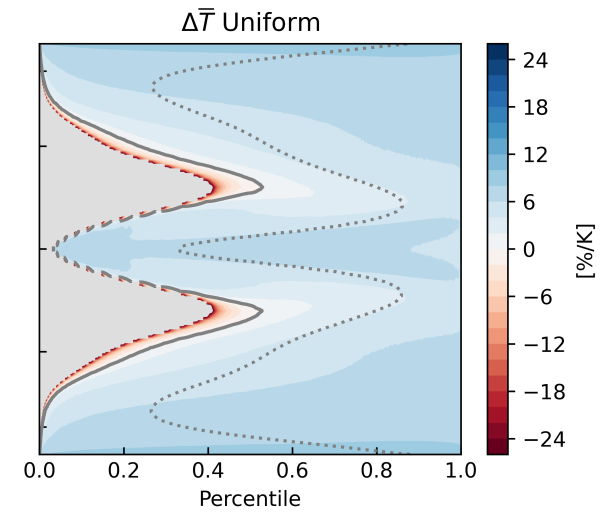

FIG. 4. Percentage change (normalized by the local zonal-mean surface temperature increase) for each daily precipitation's percentiles, for all latitudes. (a) Simulated climate change (optical thickness 1.4 times larger, warmed experiment), which includes both thermodynamic and dynamic-induced changes, (b) Thermodynamic response to the full structure of warming associated to increased optical thickness (see Fig. 1a), (c) Thermodynamic response to a vertically uniform warming with polar amplification (see Fig. 1b), and (d) Thermodynamic response to a vertically and meridionally uniform warming (see Fig. 1c). The solid grey line denotes the zero percentage change. The dotted grey line denotes the percentile at which a daily precipitation rate of $1 \mathrm{~mm} /$ day is found in the control climate (see Fig. 3); it is the same line in all four subplots. The light grey area indicates the days without rainfall $(0 \mathrm{~mm} /$ day) in the control climate (see Fig. 3).

the simulated the precipitation decrease in the subtropics (comparing $\mathrm{d}$ to $\mathrm{c}$ ), which gets further intensified by a change in the large-scale circulation (comparing $b$ to a).

\section{c. Dry extreme events: dry spells}

We analyze the changes in the statistics of the lower (dry) tail of the precipitation distribution. Rather than simply focusing on a given low percentile of daily precipitation, we consider a dry extreme index that accounts for event duration. The maximum consecutive dry days ( $\max C D D$ ) is a way to calculate the extent of dry spells in a given climate (Sillmann et al. 2013). We use a threshold of 1 mmday $^{-1}$ to separate dry days from wet days; the max CDD at each latitude is the maximum number of consecutive days with a rainfall rate under the determined threshold. Fig. $3 b$ shows the max CDD for the control simulation, which has long duration in the subtropics and high latitudes and short duration in the deep tropics and mid latitudes. The results for the changes in CDD are typically insensitive to the threshold of 1 mmday $^{-1}$, though the absolute number of CDD in the control simulation, of course, depends on the choice. Note that this index does not fully capture the whole complexity of droughts, which depend on surface changes, but is sufficient to characterize the precipitation-side of dry hydrological extremes. We examined the sensitivity of the max CDD to the GCM integration length. There were not any drastic changes when comparing a 1500 -day $(\approx 4$ years) timeseries to the 6000 -day $(\approx 16$ years $)$ shown in 
the figures. So, 6000 days is more than sufficient to assess changes in the max CDD statistic of this idealized GCM.

Figure 5a shows the simulated max CDD percentage change (dotted red line), obtained via the warmed simulation. The simulated length of dry spells decreases with warming in polar regions and the deep tropics, and increases in the subtropical regions and midlatitudes. These max CDD changes do not indicate how much more frequent dry spells are; rather, they indicate how much more intense they are. One can interpret longer dry spells as an indication of less frequent precipitation events. These general tendencies for the max CDD is in agreement with the general consensus from the IPCC 5th assessment (Sillmann et al. 2013; Orlowsky and Seneviratne 2012), and a further confirmation from the IPCC 6th assessment (Douville et al. 2021) of an intensification of wet and dry seasons under global warming such as dry spells getting drier.

The simulated thermodynamically induced changes of the max CDD (solid yellow line) is compared to the total simulated changes from the climate change simulation (dotted red line) in Figure 5a. The thermodynamic changes determined via the passive water vapor reproduce the higher latitude decrease of dry spells duration, as well as the intensification of subtropical dry spells. At higher latitudes $\left(>60^{\circ}\right)$, the max CDD decrease, about $-5 \% \mathrm{~K}^{-1}$ near the poles, is almost completely explained by the thermodynamic component. The polar regions' daily precipitation is significantly increasing at all percentiles (see Fig. $4 \mathrm{~b}$ ), where polar amplification causes a larger percentage increase in water vapor and precipitation per unit of globalmean temperature change. In the subtropics (between $10^{\circ}$ and $20^{\circ}$ ), the max CDD increases significantly, as much as $5 \% \mathrm{~K}^{-1}$. The subtropical thermodynamic intensification of dry spells is also simulated in the case of the vertically uniform thermodynamic perturbation, as shown in Figure $5 \mathrm{~b}$ (dash-dotted blue line), while the homogeneous case simulates shorter dry spells at all latitudes (dotted darkblue line). This indicates that the increased warming aloft in the tropics is not critical to explain the thermodynamic role of the subtropical dry spell intensification. The warming contrast between sea surface temperature and surface air temperature, which affects the evaporative flux of the passive water vapor, is important in simulating the amplification of dry spells in the subtropics, a region of water vapor divergence.

The implied effect of changes in the large-scale circulation (calculated as a residual in Fig. 5a, dashed orange line) further intensifies subtropical and mid-high latitudes dry spells; this agrees with an expected poleward expansion of the Hadley cell (Lau and Kim 2015) and an increase in eddy moisture flux divergence (Held and Soden 2006; Seager et al. 2010). Changes in the dry spells intensity can be linked to local changes in the moisture low-level convergence, for which a decrease would amplify the dry spell through a reduced convection in the convective zone margin [the "upped-ante mechanism", (Neelin et al. 2003; Lintner et al. 2012)].

\section{d. Wet extreme events: 99.9th percentile of daily rainfall}

We analyze the changes in the statistics of the upper (wet) tail of the precipitation distribution. Similar to many past studies (e.g., O'Gorman and Schneider 2009), we define wet extremes as events reaching a rainfall corresponding to the 99.9th percentile of the daily precipitation distribution $\left(P_{99.9}\right)$. This represents events with a return period of approximately 3 years.

Figure 6a shows the simulated $P_{99.9}$ percentage change (dotted red line), obtained via the warmed simulation. Extreme precipitation intensifies everywhere; the smallest increase (near-zero) is located in the subtropics, there is a $7-8 \% \mathrm{~K}^{-1}$ increase in the mid and high latitudes, and an increase that approaches $10 \% \mathrm{~K}^{-1}$ in the deep tropics. These simulated changes are in agreement with previously published changes (e.g., Pfahl et al. 2017).

The simulated thermodynamically induced changes of $P_{99.9}$ (solid yellow line) is compared to the total simulated changes from the climate change simulation (dotted red line) in Figure 6a. The thermodynamic changes determined via the passive water vapor reproduce the increase in heavy daily rainfall at all latitudes. At higher latitudes $\left(>50^{\circ}\right.$ ), the $P_{99.9}$ increase is almost completely explained by the thermodynamic component. The simulated percentage increase in water vapor is the largest in polar regions due to local amplified warming and increased moisture transport. Throughout the tropics, $P_{99.9}$ percentage change stays near $5 \% \mathrm{~K}^{-1}$. The $P_{99.9}$ percentage change gets larger $\left(6-7 \% \mathrm{~K}^{-1}\right)$ and more homogeneous when the thermodynamic perturbation does not include the vertical structure of warming, as shown in Figure $5 b$ (dash-dotted blue line). The surface air temperature change is around $4 \mathrm{~K}$ throughout the tropics, and is repeated through the whole atmospheric column in the "Vertically Uniform" experiment, which explains the more spatially homogeneous simulated $P_{99.9}$ changes. The contrast with the "Full Structure" simulation (Fig. 5b, solid yellow line) indicates that the upper-troposphere amplification in the tropics damps the simulated $P_{99.9}$ changes. As the climate warms, we expect to see a larger temperature increase in the uppertroposphere than at the surface in the tropics (i.e., a more stable tropical atmosphere). In our simulations, precipitation occurs immediately after condensation takes place, when the relative humidity reaches a value of $100 \%$. The large-scale condensation tendency decreases significantly in the tropical lower-troposphere because of the increasing temperature change with height. This suggests an upward shift of the level of condensation. If air parcels reach saturation at a higher altitude, where the environment temperature is much colder than at lower altitudes (higher pressures), a smaller amount of water vapor can 
Maximum Consecutive Dry Days ( $<1 \mathrm{~mm} /$ day)
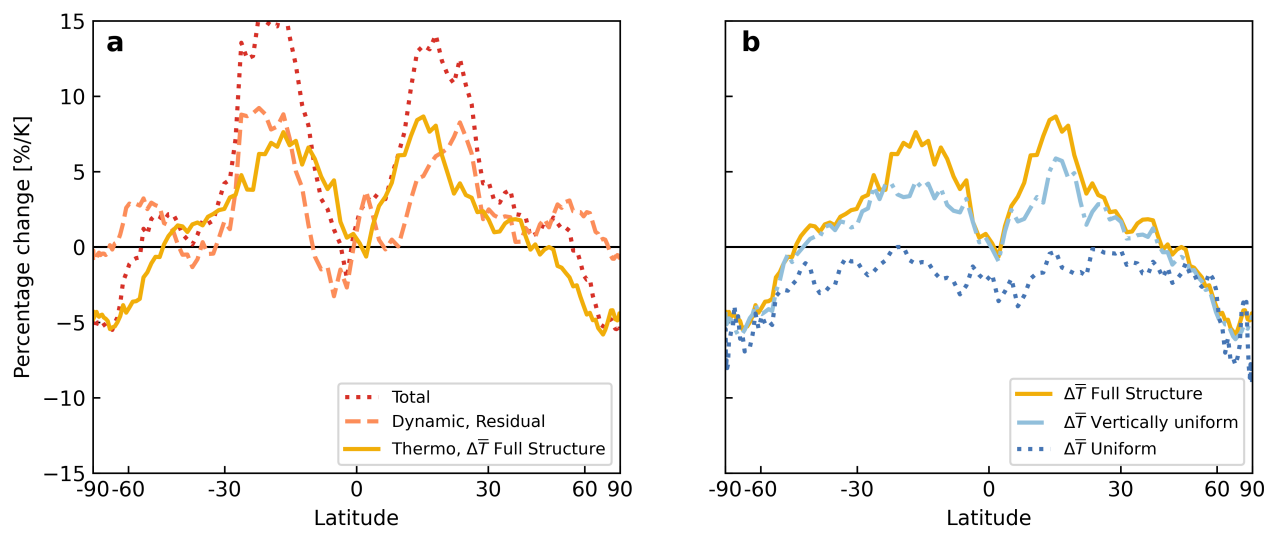

FIG. 5. Percentage change (normalized by the local zonal-mean surface temperature increase) for the maximum consecutive dry days (max CDD) at all latitudes. The threshold between dry and wet days is defined at $1 \mathrm{~mm} /$ day, following previous other studies. (a) Total changes disentangled into a thermodynamic component (from the $\Delta \bar{T}$ Full Structure experiment) and a dynamic component (residual). (b) Relative importance of the meridional structure vs the vertical structure on the dry extreme events' thermodynamic response to warming.

99.9th percentile of daily precipitation
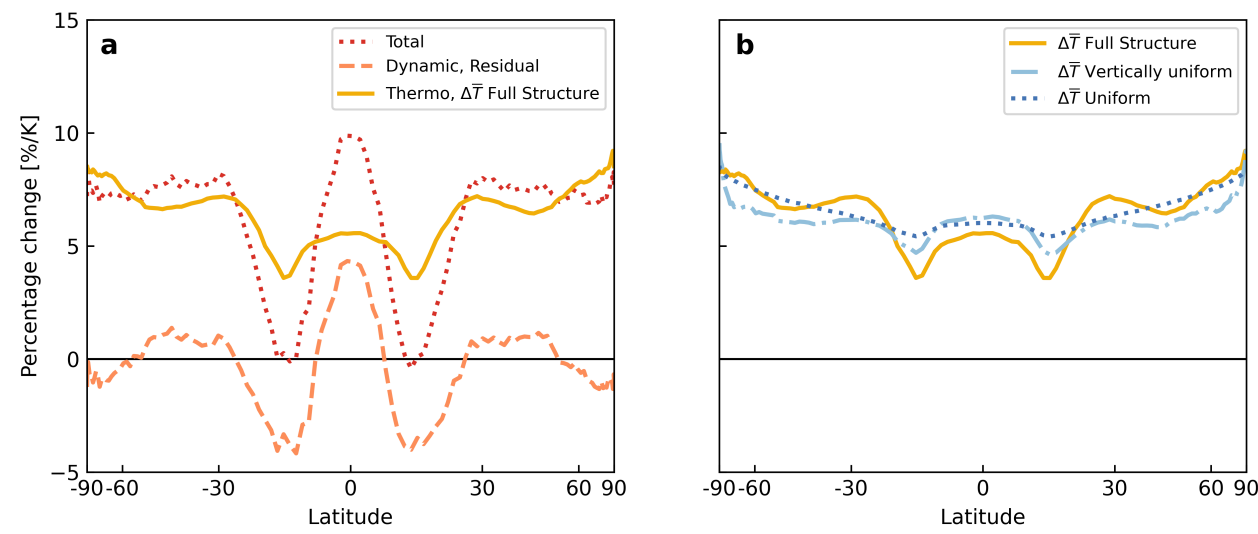

FIG. 6. Percentage change (normalized by the local zonal-mean surface temperature increase) for the 99.9 th percentile of daily precipitation at all latitudes. (a) Total changes disentangled into a thermodynamic component (from the $\Delta \bar{T}$ Full Structure experiment) and a dynamic component (residual). (b) Relative importance of the meridional structure vs the vertical structure on the wet extreme events' thermodynamic response to warming.

condense. Compared to a homogeneous warming ( 3K) perturbation (Fig. 6b, dotted dark-blue line), the $P_{99.9}$ percentage changes outside the tropics is smaller due to the normalization by the local zonal-mean surface temperature increase, which is polar amplified. One can note that the simulated $P_{99.9}$ change from the homogeneous warming experiment (dotted dark-blue line) goes from $6 \% \mathrm{~K}^{-1}$ in the deep tropics to $9 \% \mathrm{~K}^{-1}$ at the poles, which corresponds roughly to what we expect from the Clausius-Clapeyron (CC) theory (non-linear relationship between saturation specific humidity and temperature has a higher sensitivity at colder temperatures).

The implied dynamical change (Fig. 6a, residual, dashed orange line) is most important within the tropics, and it intensifies extreme precipitation in the deep tropics and weakens it in the subtropics. One can note the dominance of the thermodynamic effects over the dynamically induced changes related to strengthened/weakened vertical motion, principally outside the tropics. 


\section{e. Comparison with theory for wet extreme events}

A typical scaling for a rainfall event assumes the precipitation rate is proportional to the column-integrated condensation rate, which is expressed as the product of vertical velocity with the moist-adiabatic derivative of saturation specific humidity (Iribarne and Godson 1981):

$$
P=\left.\int_{p_{t}}^{p_{s}} \omega \frac{d q_{s}}{d p}\right|_{\theta^{*}} \frac{d p}{g},
$$

where $P$ is the surface precipitation, $p_{t}$ the tropopause pressure, $p_{s}$ the surface pressure, $\omega$ the pressure velocity, $q_{s}$ the saturation specific humidity, and $\left.\right|_{\theta^{*}}$ denotes constant equivalent potential temperature. O'Gorman and Schneider (2009) analyze extreme precipitation in a wide range of climates using this scaling for the 99.9th percentile of daily precipitation. Their analysis is based on the same idealized GCM as the present study, though they had a convection scheme. The extreme precipitation scaling is evaluated using the pressure velocity, temperature, and surface pressure variables conditioned on the $P_{99.9}$ extreme events occurring. One can decompose changes in the perturbed scaling into dynamic and thermodynamic components:

$$
\delta P=\delta\left\{\left.\omega_{e} \frac{d q_{s}}{d p}\right|_{\theta^{*}, T_{e}}\right\}=\left\{\left.\delta\left(\omega_{e}\right) \frac{d q_{s}}{d p}\right|_{\theta^{*}, T_{e}}+\left.\omega_{e} \frac{d q_{s}}{d p}\right|_{\theta^{*}, T_{e}+\Delta T}\right\}+\text { residual }
$$

where $\omega_{e}$ and $T_{e}$ are respectively the pressure velocity and temperature conditioned on an extreme rainfall event, and $\{\cdot\}$ is the vertical mass-weighted integral.

In Figure 7, we present the same decomposition of the 99.9th percentile of daily precipitation response to warming as in the previous section but evaluated with the scaling (equation 4). The total percentage change (dotted red line) is calculated by taking the difference between the $P_{99.9}$ scaling evaluated for the control and the warmed experiments.

We evaluate the thermodynamic component of the scaling (solid grey line) by keeping the pressure velocity fixed. The moist-adiabatic derivative of saturation specific humidity term is perturbed by a change in temperature. Rather than using the changes in the conditioned temperature on $P_{99.9}\left(\Delta T_{e, 99.9}\right)$ to evaluate the scaling thermodynamic component, we use the changes in the time-mean, zonal-mean temperature $(\Delta \bar{T})$, similar to the passive water vapor "Full Structure" experiment (see Fig. 1a). As discussed by O'Gorman and Schneider (2009), the temperature profile when the extreme event occurs is warmer than the time-mean temperature perturbation. When we evaluate the scaling thermodynamic component using $\Delta T_{e, 99.9}$, predicted changes are systematically lower than when using $\Delta \bar{T}$ as the perturbation, with larger discrepancies outside the tropics.

We compare the thermodynamically induced changes simulated via the passive experiment "Full Structure" (Fig. 7 , solid yellow line) with the thermodynamic component of the scaling (Fig. 7, solid grey line). They both show a large sensitivity of $P_{99.9}$ to warming increasing poleward the lowest sensitivity located in the deep tropics. They differ in the higher latitudes $\left(>50^{\circ}\right)$, where the passive framework predicts a large sensitivity of the $P_{99.9}$ thermodynamic component to warming and a near zero or somewhat negative contribution by the dynamic component.
The scaling explains the thermodynamically induced effects on extreme precipitation via the changes in the vertical gradient of saturation specific humidity along a moistadiabat. They assume an atmosphere in a moist adiabatic state when $P_{99.9}$ extreme events occur, which moderates the changes of saturation specific humidity increases with height. For weaker changes in the vertical gradient of saturation specific humidity, less condensation occurs with the same vertical velocity. We can make a similar assumption, one of an atmosphere with a saturated moist-adiabatic lapse rate, for the passive water vapor GCM experiment "MoistAdiabat", described in section 2c (see Fig. 1d). In this experiment, the upper-troposphere warms more than the surface everywhere. The simulated thermodynamically induced $P_{99.9}$ changes based on a moist-adiabat profile are compared to the scaling thermodynamic component predictions in Fig. 7 (dot-dashed blue line versus solid grey line). The magnitude of differences between passive water vapor GCM estimate of the thermodynamic changes and the scaling theory contrast is significantly reduced. The moist adiabatic temperature change reduces the $P_{99.9}$ percentage change with warming, in the deep tropics and midlatitudes. In the previous subsection, we mentioned how the increasing temperature change with height moderates the $P_{99.9}$ sensitivity relative to surface warming. The role of vertical structure of warming is similar here.

We evaluate the dynamic component of the scaling (dashed orange line) by keeping fixed the temperature, which means there are no changes in the moist-adiabatic derivative of saturation specific humidity term. Similarly to previous results, changes in the pressure velocity statistics have a significant impact on the predicted changes in extreme precipitation intensity. There is a dynamical strengthening of extreme precipitation at higher latitudes that one could explained through the theory of the stormtrack's poleward shift in a warmer climate and also, possi- 
99.9th percentile of daily precipitation (Scaling vs Passive experiments)

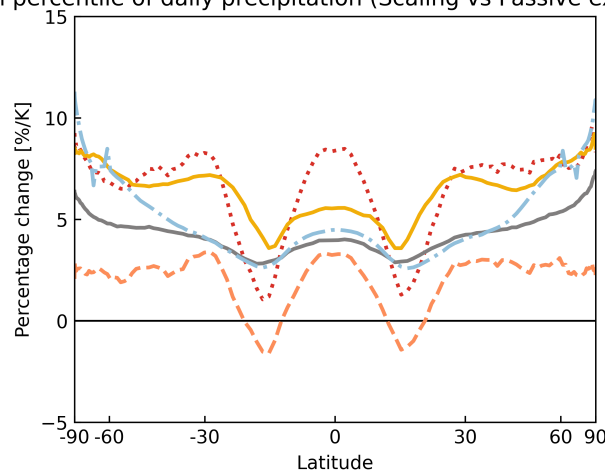

.... Total, $\left[\delta\left(\omega \partial q_{s} / \partial p\right)\right]$

- - Dynamic, $\left[\delta(\omega) \partial q_{s} / \partial p\right]$

- Thermo, $\left[\omega \delta\left(\partial q_{s} / \partial p\right)\right]$ with $\Delta \bar{T}$ Full Structure

- Thermo, Passive with $\Delta \bar{T}$ Full Structure

- Thermo, Passive with $\Delta \bar{T}$ Moist Adiabat

Fig. 7. Percentage change (normalized by the local zonalmean surface temperature increase) for the 99.9th percentile of daily precipitation at all latitudes. Similar to Figure 6a, but here the total changes, thermodynamic and dynamic components are obtained via the scaling from OG09 (see equation 3). The total percentage change is evaluated with surface pressure, pressure velocity and temperature profiles conditioned on the extremes occurring, taken from the control and warmed simulations. The dynamic component of the scaling is evaluated with fixed temperature and changing pressure velocity. The thermodynamic component of the scaling is evaluated with fixed pressure velocity and temperature profile perturbed by the timemean, zonal-mean $\Delta \bar{T}$ from "Full Structure" experiment. The latter is compared to the thermodynamic component obtained via the passive water vapor, for both the $\Delta \bar{T}$ "Full Structure" and $\Delta \bar{T}$ "Moist-adiabat" experiments (see Fig. 1a \& d).

bly, an intensification. There is also a significant dynamical strengthening effect in the deep tropics, which depends on the choice to forgo a convection parameterization (cf. Fig. $8 \mathrm{~b}$ of O' Gorman and Schneider 2009). We note that this estimate of the dynamic changes to precipitation extremes is higher at most latitudes than the residual calculation shown in Fig. 6a. These dynamic changes differ partly because of the discrepancies between the thermodynamic changes obtained via the passive water vapor and via the scaling. Since the dynamic changes in Fig. 6a is simply calculated as a residual, the fact that the estimates of the thermodynamic changes differ (solid yellow line compared to solid grey line in Fig. 7) is the main factor that leads to large differences for the dynamic component to extreme precipitation changes. Hence, calculating the residual from the thermodynamic changes with moist-adiabatic temperature change (Fig. 7, dot-dashed blue line) would reduce the differences between the estimates of the dynamic changes (not shown). Also, there are differences in the assump- tions made by the scaling and by our current approach that could impact the results (e.g., the scaling does not include horizontal advection of water vapor or evaporation in its approximation to the atmospheric water vapor budget).

\section{Summary and discussion}

We performed an online-calculation of the thermodynamic precipitation changes using an additional passive water vapor variable that undergoes evaporation and condensation at a climate change-perturbed temperature. This methodology can be applied both to individual storms (Fig. 2) and across the whole probability distribution of daily rainfall (Fig. 4). We simulate a thermodynamically induced precipitation rate decrease in the subtropics at almost all percentiles (all dry and most wet days). In the midlatitudes, there is a thermodynamically induced precipitation rate decrease at lower percentiles (dry days), which is further amplified by dynamical changes. With this approach, we can identify which aspects of the temperature change structure are important to simulating particular aspects of the changes in the precipitation distribution.

The dry tail of the daily precipitation distribution can be described using the maximum consecutive dry day (CDD) index, a measure of dry spell length. We demonstrated that there is a thermodynamically induced intensification of dry spells in the subtropics, which is further amplified by dynamical changes. Most latitudes with a simulated daily precipitation rate decrease for dry days (percentiles with rainfall lower than the $1 \mathrm{~mm} /$ day threshold, red contours to the left of the gray dotted line in Fig. 4) correspond to the latitudes with longer dry spells (Fig. 5). The subtropical thermodynamically induced intensification of dry spells is simulated when the warming difference between sea surface temperature and surface air temperature is included. The reduction in this temperature contrast affects the evaporative flux here and in comprehensive simulations of climate change (e.g., Richter and Xie 2008). We show that the thermodynamic changes of CDD has a nearly CC increase in the subtropics and decreases in high latitudes. Previous thermodynamic discussions comparing the mean hydrological cycle response to global warming to wet extremes changes have been able to give information on the sign of dry extremes changes but not on their magnitude. We have a unique quantitative approach that allows us to actually quantify the magnitude of changes for dry spells.

The upper, wet tail of the daily precipitation distribution can be described using the 99.9th percentile of daily precipitation, a heavy rainfall event. We demonstrate that the thermodynamically induced increase in extreme precipitation dominates over dynamical effects outside the tropics. We show a damping of the extreme precipitation intensification from the moist-adiabatic warming in the upper-troposphere. We can interpret this as the result of weakening vertical gradients in the saturation specific 
humidity, consistent with previous theory for precipitation extremes.

This passive water vapor, on-line approach can be extended to additional categories of extreme events and comprehensive models. We are pursuing the analysis of simulations that contain a large number of tropical cyclones. Adapting comprehensive GCMs with isotope-enabled versions could also be used to examine climate change's effect on precipitation, rather than the fractionation of heavy isotopes. In addition, this methodological approach can be used to understand mean precipitation changes under global warming, including assessments of the role of the vertical and spatial distribution of warming, which we examine in forthcoming research.

Acknowledgments. We acknowledge the support of an NSERC Discovery grant and Canada Research Chair (Tier II) and the Canada Foundation for Innovation for a Compute Canada allocation. We thank Isaac Held for helpful discussions.

Data availability statement. The GCM source code for the passive water vapor version of the idealized GCM is available at https://github.com/marielabonte/GCMpassive-hydro.git .

\section{References}

Abbott, T. H., T. W. Cronin, and T. Beucler, 2020: Convective dynamics and the response of precipitation extremes to warming in radiativeconvective equilibrium. J. Atmos. Sci., 77 (5), 1637-1660.

Allen, M. R., and W. J. Ingram, 2002: Constraints on future changes in climate and the hydrologic cycle. Nature, 419 (6903), 228.

Bosilovich, M. G., and S. D. Schubert, 2002: Water vapor tracers as diagnostics of the regional hydrologic cycle. J. Hydrometeor., 3 (2), $149-165$.

Chen, G., Y. Ming, N. D. Singer, and J. Lu, 2011: Testing the ClausiusClapeyron constraint on the aerosol-induced changes in mean and extreme precipitation. Geophys. Res. Lett., 38 (4).

Chen, G., J. Norris, J. D. Neelin, J. Lu, L. R. Leung, and K. Sakaguchi, 2019: Thermodynamic and dynamic mechanisms for hydrological cycle intensification over the full probability distribution of precipitation events. J. Atmos. Sci., 76 (2), 497-516.

Colose, C. M., A. N. LeGrande, and M. Vuille, 2016: The influence of volcanic eruptions on the climate of tropical South America during the last millennium in an isotope-enabled general circulation model. Climate of the Past, 12 (4), 961-979.

Dai, A., T. Zhao, and J. Chen, 2018: Climate change and drought: a precipitation and evaporation perspective. Current Climate Change Reports, 4 (3), 301-312.

Douville, H., and Coauthors, 2021: Water cycle changes. Climate Change 2021: The Physical Science Basis. Contribution of Working Group I to the Sixth Assessment Report of the Intergovernmental Panel on Climate Change, V. Masson-Delmotte, P. Zhai, A. Pirani, S. Connors, C. Péan, S. Berger, N. Caud, Y. Chen, L. Goldfarb,
M. Gomis, M. Huang, K. Leitzell, E. Lonnoy, J. Matthews, T. Maycock, T. Waterfield, O. Yelekçi, R. Yu, and B. Zhou, Eds., Cambridge University Press.

Emanuel, K., 2017: Assessing the present and future probability of Hurricane Harvey's rainfall. Proc. Natl. Acad. Sci. (USA), 114 (48), 12681-12684.

Fischer, E. M., U. Beyerle, and R. Knutti, 2013: Robust spatially aggregated projections of climate extremes. Nature Climate Change, 3 (12), 1033.

Frierson, D. M., 2007: The dynamics of idealized convection schemes and their effect on the zonally averaged tropical circulation. J. Atmos. Sci., 64 (6), 1959-1976.

Frierson, D. M., I. M. Held, and P. Zurita-Gotor, 2006: A gray-radiation aquaplanet moist GCM. Part I: Static stability and eddy scale. $J$. Atmos. Sci., 63 (10), 2548-2566.

Galewsky, J., A. Sobel, and I. Held, 2005: Diagnosis of subtropical humidity dynamics using tracers of last saturation. J. Atmos. Sci., 62 (9), 3353-3367.

Grabowski, W. W., 2014: Extracting microphysical impacts in largeeddy simulations of shallow convection. J. Atmos. Sci., 71 (12), $4493-$ 4499 .

Grabowski, W. W., 2015: Untangling microphysical impacts on deep convection applying a novel modeling methodology. J. Atmos. Sci., 72 (6), 2446-2464.

Held, I. M., 2005: The gap between simulation and understanding in climate modeling. Bull. Amer. Meteor. Soc., 86, 1609-1614.

Held, I. M., and B. J. Soden, 2006: Robust responses of the hydrological cycle to global warming. J. Climate, 19 (21), 5686-5699.

Held, I. M., and M. J. Suarez, 1994: A proposal for the intercomparison of the dynamical cores of atmospheric general circulation models. Bull. Amer. Meteor. Soc., 75 (10), 1825-1830.

Iribarne, J., and W. Godson, 1981: Atmospheric Thermodynamics. 2nd ed., D. Reidel Publishing Company.

Jeevanjee, N., P. Hassanzadeh, S. Hill, and A. Sheshadri, 2017: A perspective on climate model hierarchies. J. Adv. Model. Earth Syst., $1760-1771$.

Lackmann, G. M., 2015: Hurricane Sandy before 1900 and after 2100. Bull. Amer. Meteor. Soc., 96, 547-560.

Lau, W. K., and K.-M. Kim, 2015: Robust Hadley circulation changes and increasing global dryness due to $\mathrm{CO} 2$ warming from CMIP5 model projections. Proc. Natl. Acad. Sci. (USA), 112 (12), 36303635 .

Lee, J.-E., K. Johnson, and I. Fung, 2009: Precipitation over South America during the Last Glacial Maximum: An analysis of the "amount effect" with a water isotope-enabled general circulation model. Geophys. Res. Lett., 36 (19).

Li, Z., and P. A. O'Gorman, 2020: Response of vertical velocities in extratropical precipitation extremes to climate change. J. Climate, 33 (16), 7125-7139.

Lintner, B. R., M. Biasutti, N. S. Diffenbaugh, J.-E. Lee, M. J. Niznik, and K. L. Findell, 2012: Amplification of wet and dry month occurrence over tropical land regions in response to global warming. $J$. Geophys. Res.: Atmos., 117 (D11). 
Lu, J., L. Ruby Leung, Q. Yang, G. Chen, W. D. Collins, F. Li, Z. Jason Hou, and X. Feng, 2014: The robust dynamical contribution to precipitation extremes in idealized warming simulations across model resolutions. Geophys. Res. Lett., 41 (8), 2971-2978.

Maher, P., and Coauthors, 2019: Model hierarchies for understanding atmospheric circulation. Rev. Geophys., 57, 250-280.

Ming, Y., and I. M. Held, 2018: Modeling water vapor and clouds as passive tracers in an idealized GCM. J. Climate, 31 (2), 775-786.

Muller, C. J., P. A. O'Gorman, and L. E. Back, 2011: Intensification of precipitation extremes with warming in a cloud-resolving model. $J$. Climate, 24 (11), 2784-2800.

Neelin, J., C. Chou, and H. Su, 2003: Tropical drought regions in global warming and El Niño teleconnections. Geophys. Res. Lett., 30 (24).

Nie, J., P. Dai, and A. H. Sobel, 2020: Dry and moist dynamics shape regional patterns of extreme precipitation sensitivity. Proc. Natl. Acad. Sci. (USA), 117 (16), 8757-8763.

Norris, J., G. Chen, and C. Li, 2020: Dynamic amplification of subtropical extreme precipitation in a warming climate. Geophys. Res. Lett., 47 (14), e2020GL087 200.

Orlowsky, B., and S. I. Seneviratne, 2012: Global changes in extreme events: regional and seasonal dimension. Climatic Change, 110 (3-4), 669-696.

O'Gorman, P. A., and T. Schneider, 2008: The hydrological cycle over a wide range of climates simulated with an idealized GCM. J. Climate, 21 (15), 3815-3832.

O'Gorman, P. A., and T. Schneider, 2009: Scaling of precipitation extremes over a wide range of climates simulated with an idealized GCM. J. Climate, 22 (21), 5676-5685.

Pall, P., M. Allen, and D. A. Stone, 2007: Testing the Clausius-Clapeyron constraint on changes in extreme precipitation under $\mathrm{CO} 2$ warming. Climate Dyn., 28 (4), 351-363.

Pfahl, S., P. A. O'Gorman, and E. M. Fischer, 2017: Understanding the regional pattern of projected future changes in extreme precipitation. Nature Climate Change, 7 (6), 423.

Reed, K. A., A. Stansfield, M. Wehner, and C. Zarzycki, 2020: Forecasted attribution of the human influence on Hurricane Florence. Science advances, 6 (1), eaaw9253.

Richter, I., and S.-P. Xie, 2008: Muted precipitation increase in global warming simulations: A surface evaporation perspective. J. Geophys. Res., 113, D24 118.

Seager, R., N. Naik, and G. A. Vecchi, 2010: Thermodynamic and dynamic mechanisms for large-scale changes in the hydrological cycle in response to global warming. J. Climate, 23 (17), 4651-4668.

Sillmann, J., V. V. Kharin, F. Zwiers, X. Zhang, and D. Bronaugh, 2013: Climate extremes indices in the CMIP5 multimodel ensemble: Part 2. Future climate projections. J. Geophys. Res.: Atmos., 118 (6), 2473-2493.

Tandon, N. F., X. Zhang, and A. H. Sobel, 2018: Understanding the dynamics of future changes in extreme precipitation intensity. Geophys. Res. Lett., 45 (6), 2870-2878.

Trenberth, K. E., A. Dai, R. M. Rasmussen, and D. B. Parsons, 2003: The changing character of precipitation. Bull. Amer. Meteor. Soc., 84 (9), 1205-1218.
Trenberth, K. E., C. A. Davis, and J. Fasullo, 2007: Water and energy budgets of hurricanes: Case studies of Ivan and Katrina. J. Geophys. Res.: Atmos., 112 (D23). 